\title{
Polyglycerol Fatty Acid Ester Based Lipid Nanoparticles: Physical Stability and Crystalline Status
}

\author{
$\underline{\text { A. KovaceVIC }}^{1}$, S. Savic ${ }^{1}$, J. Milic ${ }^{1}$, R. H. MÜLleR ${ }^{2}$, C. M. KeCK ${ }^{2,3}$ \\ ${ }^{1}$ Department of Pharmaceutical Technology and Cosmetology, Faculty of Pharmacy, University of Belgrade, \\ Serbia \\ ${ }^{2}$ Department of Pharmaceutics, Biopharmaceutics \& NutriCosmetics, Free University of Berlin, Germany \\ ${ }^{3}$ Fachhochschule Kaiserslautern, University of Applied Sciences Kaiserslautern, Angewandte Logistik- und \\ Polymerwissenschaften Pirmasens, Carl-Schurz-Straße 10-16, 66953 Pirmasens, Germany \\ E-mails: andjelkakovacevic@yahoo.com (A. Kovacevic), nanoteam@gmx.com (R. H. Müller),
}

Sci Pharm. 2010; 78: 681

doi:10.3797/scipharm.cespt.8.PNM11

Formulation development of nanostructured lipid carriers (NLC) aims physical stability in both particle size and crystalline state. Besides production parameters, the selection of appropriate stabilizers is the most crucial parameter in the development. Very often the influence of different stabilizers on the size and the physical stability is investigated. However, little is known about the influence of stabilizers on the crystalline state of lipid nanoparticles (LN). Thus, two surfactants of polyglycerol fatty acid ester type were screened for their ability to form physically stable NLC and for their influence on the crystalline state of the lipid matrix. The investigated surfactants are polyglycerol 6-distearate (PD) and polyglycerol 6-isostearate (PI). Physical stability study was performed using light microscopy, dynamic and low angle static light scattering. DSC study was used to analyze the crystalline status of LN. Both surfactants in the concentration of $1.0 \%(\mathrm{w} / \mathrm{w})$ led to almost similar $L N$ with a mean diameter between 180-200 nm. LN were stable over the period of 90 days. The absence of large particles was also confirmed by light microscopy and low angle static light scattering. In contrast to this significant differences were found within DSC thermograms. LN stabilized with PD show a melting event, whereas LN stabilized with PI show no melting event. Hence, PI inhibited the crystallization of the lipid matrix, leading to a non-crystalline LN instead of NLC. A possible explanation is that PD which has a solid lipid tail leads to a different interaction with lipid matrix, than the liquid lipid tail surfactant (PI). These interactions can lead either to an initiated crystallization or to the circumvention of crystallization. In conclusion: Stabilizers can influence the crystalline state of the lipid matrix of LN. Thus, for a successful development, this parameter needs to be investigated. Furthermore, it might be a helpful tool to develop NLC with a "tailor-made" crystalline state and release profile in the future.

The authors would like to acknowledge the financial support obtained from DAAD (Deutscher Akademischer Austauschdienst) for the present research. 